\title{
Method of Nonperturbing Measurements of the Electron Bunch Length Based on Coherent Diffraction Radiation
}

\author{
G. A. Naumenko ${ }^{a, *}$, A. P. Potylitsyn ${ }^{a}$, M. V. Shevelev ${ }^{a}$, D. A. Shkitov ${ }^{a}$, and Yu. A. Popov ${ }^{b}$ \\ ${ }^{a}$ Tomsk Polytechnic University, Tomsk, 634004 Russia \\ ${ }^{b}$ Joint Institute for Nuclear Research, Moscow oblast, Dubna, 141980 Russia \\ *e-mail: naumenko@tpu.ru
}

Received November 1, 2020; revised November 18, 2020; accepted November 18, 2020

\begin{abstract}
A simple nonperturbing technique for the electron relativistic bunch diagnostics has been proposed which does not require the use of an interferometer for spectral measurements. The interferometer functions are performed by a slit target in which diffraction radiation is generated. The measurements of electron bunch length are carried out by shifting one half of the target relative to the other. A good agreement with the results of measuring the coherent radiation spectrum of relativistic electron bunches has been obtained.
\end{abstract}

Keywords: relativistic electrons, beam parameters, technique of measurements

DOI: $10.1134 /$ S1547477121020126

\section{INTRODUCTION}

The efficiency of operating many facilities, such as free electron lasers and plasma-wave accelerators, is determined not only by minimizing the transverse beam dimensions, but also by minimizing the longitudinal dimensions of electron bunches. For this purpose, a variety of compressors of longitudinal size of electron bunches is used. In this case, the control of the longitudinal size and the profile of electron bunches is no less important. Streak cameras with picosecond resolution are not only expensive and difficult to operate, but also have fundamental limitations when improving resolution. Alternative methods are based on measuring the spectrum of coherent radiation of various types: transition [1] or synchrotron radiation [2].

The manifestation of coherent effects in most cases depends on the ratio of the electron bunch length to the wavelength of radiation under study. This dependence can be used in measuring the electron bunch length. For this purpose, it is sufficient to have a spectral distribution of radiation in the wavelength range, including the expected length of electron bunches. In general, calculating the longitudinal profile of an electron bunch from the spectral distribution of radiation is an inverse Fredholm problem of first kind, which has very a poor convergence for real conditions. Therefore, as a rule, an assumption is made about the longitudinal profile of an electron bunch (most often a Gaussian) with one parameter, and the value of the parameter is found by fitting the model dependence to the measured spectrum. Such measurements using various types of radiation are becoming traditional.

Measurements of coherent spectra are carried out using various spectrometers, such as spectrometers based on diffraction gratings [3], Michelson interferometers [4, 5], polychromators [6], and MartinPuplett interferometers [7]. All the abovementioned spectrometers are rather cumbersome and difficult to set up; therefore, their use for monitoring and controlling the beam parameters is very problematic.

In 1997, independently, Lampel [8] and Nguyen [9] proposed the idea of using Smith-Purcell radiation (SPR) as a diagnostic tool for picosecond bunches. As was noted in [10], they noticed that SPR will be generated at large angles relative to the beam propagation, and its intensity will be much higher than the intensity of incoherent SPR. This property of SPR was noted already in 1953 [11]. One feature of the approach in $[8,9]$ is that the proposed diagnostics is nonperturbing. As for the rest of the details, the approach in these works is not much different from the approaches using coherent transition or diffraction radiation (for example, in [3]), in which the radiation spectrum measurement is a separate problem. In [12], we tested a technique for measuring the length of electron bunches based on coherent Smith-Purcell radiation, in which the periodic target itself was used as a spectrometer without the use of external spectrometric equipment. This geometry made it possible to significantly simplify the scheme for measuring the length of electron bunches. However, the experience 
of using Smith-Purcell radiation for these purposes showed its essential drawback. Namely, the intensity of Smith-Purcell radiation is significantly lower than the intensity of transition and diffraction radiation, which reduces the sensitivity of the method and narrows the area of its application.

In [13], we considered the possibility of implementing a simple scheme of nonperturbing diagnostics based on diffraction radiation, in which the diffraction radiation target itself acts as an interferometer for measuring the length of electron bunches. Below, we present a description of this technique and the experimental results of its application using an alternative approach to choose a criterion for estimating the electron bunch length.

\section{METHOD DESCRIPTION}

Let us consider the scheme of diffraction radiation of relativistic electrons on a slit target (Fig. 1) when electrons move along the $z$ axis, flying through the slit, and generate diffraction radiation from both halfplanes. The target turned relative to the $z$ axis at angle $\psi$ (usually $\psi=45^{\circ}$ ); the $y^{\prime}$ axis is directed along the direction of specular reflection. When $\psi=45^{\circ}$, the $y$ and $y^{\prime}$ axes coincide.

In the far zone, the diffraction radiation-field strength of relativistic electrons from parallel halfplanes can be calculated using the expression from [14]. Here we are only interested in the field vector component in the $y^{\prime} z$ plane (in terms of Fig. 1) for radiation from a half-plane.

$$
\begin{gathered}
E_{x}=i \frac{e}{2 \pi} \frac{\theta_{x}}{\sqrt{1+\theta_{x}^{2}}\left(\sqrt{1+\theta_{x}^{2}}+i \theta_{y}\right)} \\
\times \exp \left\{-\omega \frac{a}{2 \gamma}\left(\sqrt{1+\theta_{x}^{2}}+i \theta_{y}\right)\right\}, \\
E_{y}=-\frac{e}{2 \pi} \frac{1}{\sqrt{1+\theta_{x}^{2}}+i \theta_{y}} \exp \left\{-\omega \frac{a}{2 \gamma}\left(\sqrt{1+\theta_{x}^{2}}+i \theta_{y}\right)\right\},
\end{gathered}
$$

where $a$ is the gap thickness; $\gamma$ is the Lorentz factor; and $\theta_{x}$ and $\theta_{y}$ are the observation angles relative to the direction of specular reflection, normalized to the angle $1 / \gamma$. If one half of the target is shifted in the $z$ direction by distance $d$, the total field strength of the detected radiation and its intensity will depend on the phase $\varphi=\frac{\omega d}{c}$ :

$$
\begin{gathered}
E_{x s l}^{d}=E_{x}-E_{x}^{*} \exp \left(-i \frac{\omega d}{c}\right), \\
E_{y s l}^{d}=E_{y}-E_{y}^{*} \exp \left(-i \frac{\omega d}{c}\right), \\
\frac{d W}{d \omega d \Omega}=\mathrm{const}\left(\left|E_{x s l}^{d}\right|^{2}+\left|E_{y s l}^{d}\right|^{2}\right)=W_{0}(d) .
\end{gathered}
$$

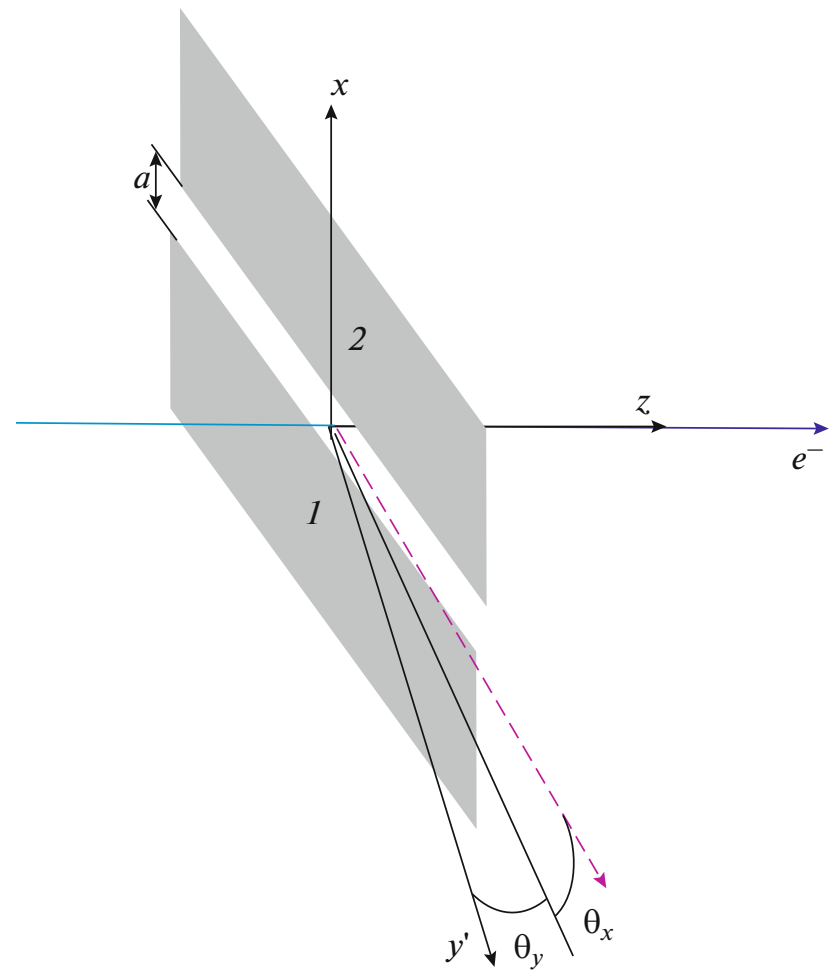

Fig. 1. Scheme of diffraction radiation of relativistic electrons on a slit target

After integration over detector aperture $W_{1}=\int W_{0} d \theta x d \theta y$ and over circular frequency $\omega$, we have

$$
W_{2}=\int_{\omega_{\min }}^{\omega_{\max }} W_{1} \exp \left(-(\omega \sigma)^{2}\right) \operatorname{eff}(\omega) d \omega,
$$

where eff $(\omega)$ is the detector spectral intensity, the exponential factor describes the longitudinal formfactor of an electron bunch in the approximation of its large population, and $\sigma$ is the rms deviation of the electron bunch.

This expression can be used to plot a theoretical interferogram (dependence on $d$ ) for each value of the electron bunch length $\sigma$ for specific experimental conditions.

For further calculations, we pass in (1) to relative quantities in the system of units in which the speed of light is $\mathrm{C}=1$ :

$$
\begin{gathered}
d_{-}=d / \sigma, \quad a_{-}=a / \sigma, \omega_{1-}=\omega_{\min } \sigma, \\
\omega_{2_{-}}=\omega_{\max } \sigma, W_{2_{-}}=W_{2} \sigma .
\end{gathered}
$$

In this representation, interferograms do not depend on length $\sigma$ of electron bunches.

The most significant parameter is the limitation of the detector's efficiency in the short wavelength range $\omega_{2}$. The calculated interferogram is shown in Fig. 2. 


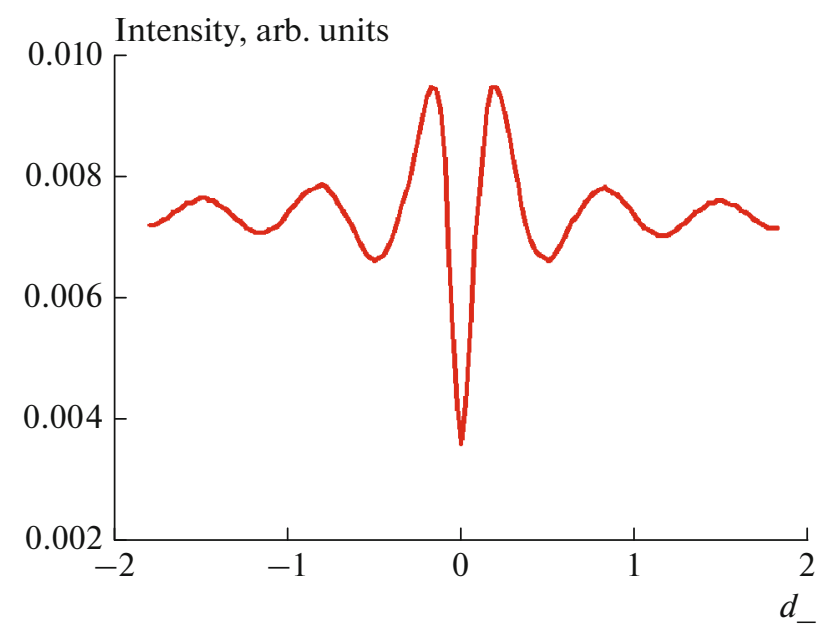

Fig. 2. Calculated interferogram for $a_{-}=0.01, \gamma=12$, $\Delta \theta=0.1, \omega_{1_{-}}=0.5$ and $\omega_{2}=2$.

In principle, it is possible to use the position of the first maximum or the intensity ratios of the first maximum and subsequent ones as a criterion for determining the length of an electron bunch having a length of several $\mathrm{mm}$. This approach was used in [13], which provided a change in the criterion by less than $2.4 \%$ when the electron bunch length changed by $1 \mathrm{~mm}$.

The analysis showed that the optimal criterion providing the highest sensitivity for measuring the electron bunch length is the position of maximum $d_{-\max }$ of the interferogram derivative with respect to parameter $d_{-}$. In this representation, we have $\sigma=\frac{d_{\max }}{d_{-\max }}$, where $d_{\max }$ is the derivative maximum position in the measured interferogram and $d_{-\max }$ is the theoretical derivative maximum position in rela-

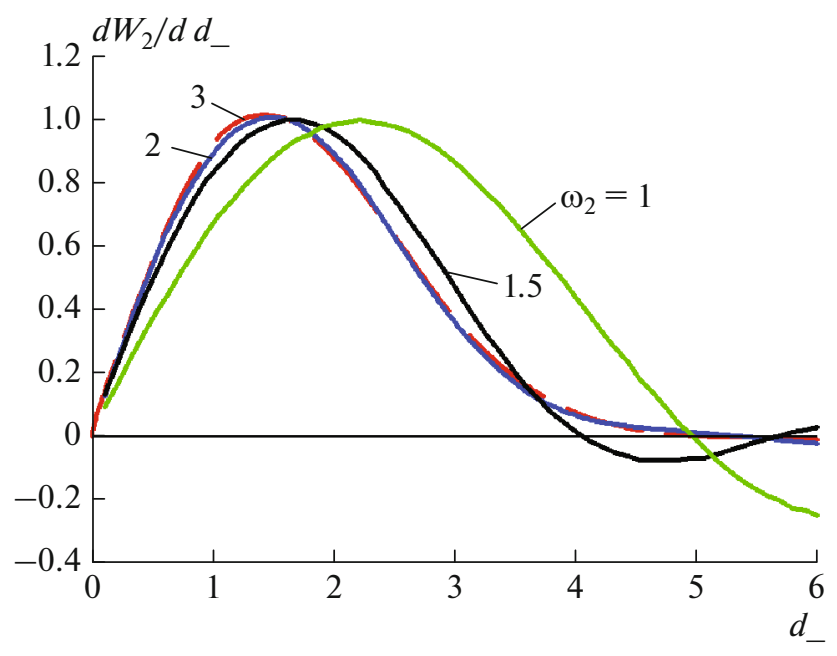

Fig. 3. Interferogram derivative for $a_{-}=0.01 ; \gamma=12$; $\Delta \theta=0.1 ; \omega_{1_{-}}=0.1 ;$ and $\omega_{2}=3,2,1.5,1$. tive units of $d_{-}$. In this case, it is necessary to determine the frequency range of the detector sensitivity, which is necessary to ensure maximum sensitivity when measuring the electron bunch length. Figures 3 and 4 show the derivatives of interferograms with

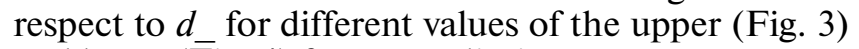
and lower (Fig. 4) frequency limits.

Figures 3 and 4 show that the optimal frequency range for measuring the length of electron bunches lies within the limits

$$
\begin{aligned}
\omega_{-}=\omega \sigma & =0.5 \sim 2, \\
\text { or frequency range } v_{\mathrm{GHz}} & =\frac{300}{2 \pi \sigma_{\mathrm{mm}}}(0.5 \sim 2)
\end{aligned}
$$

For example, for $\sigma=0.8 \mathrm{~mm}, v_{\mathrm{GHz}}=30-90 \mathrm{GHz}$,

$$
\text { for } \sigma=0.5 \mathrm{~mm}, v_{\mathrm{GHz}}=75-140 \mathrm{GHz} \text {, }
$$$$
\text { for } \sigma=4 \mathrm{~mm} \mathrm{v}_{\mathrm{GHz}}=6-24 \mathrm{GHz} \text {. }
$$

The problem is that, in order to determine the optimal limits of integration over $\omega$ using (2) in real measurements of the electron bunch lengths, the value of $\sigma$ is not yet known and the limits of integration are compared with the limits of the detector spectral sensitivity, which are determined by the choice of the detector and may not be optimal. In this case, a priori information is needed to estimate the electron bunch length (for example, using characteristics of the microwave system of the accelerator). The effect of slit width $a$ on the position of the interferogram derivative maximum, shown in Fig. 5, turns out to be insignificant up to $a=10 \sigma$.

\section{EXPERIMENT}

\subsection{Electron Beam Parameters and Setup Layouts}

The experiments on measuring the length of electron bunches were carried out on a microtron beam

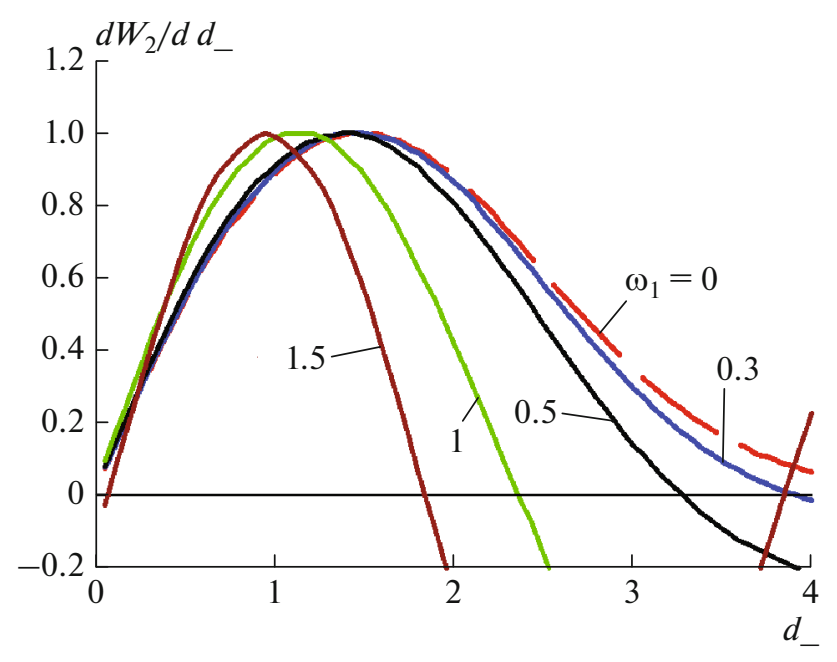

Fig. 4. Interferogram derivative for $a_{-}=0.01 ; \gamma=12$; $\Delta \theta=0.1 ; \omega_{2}=2$; and $\omega_{1_{-}}=0,0.3,0.5,1,1.5$. 


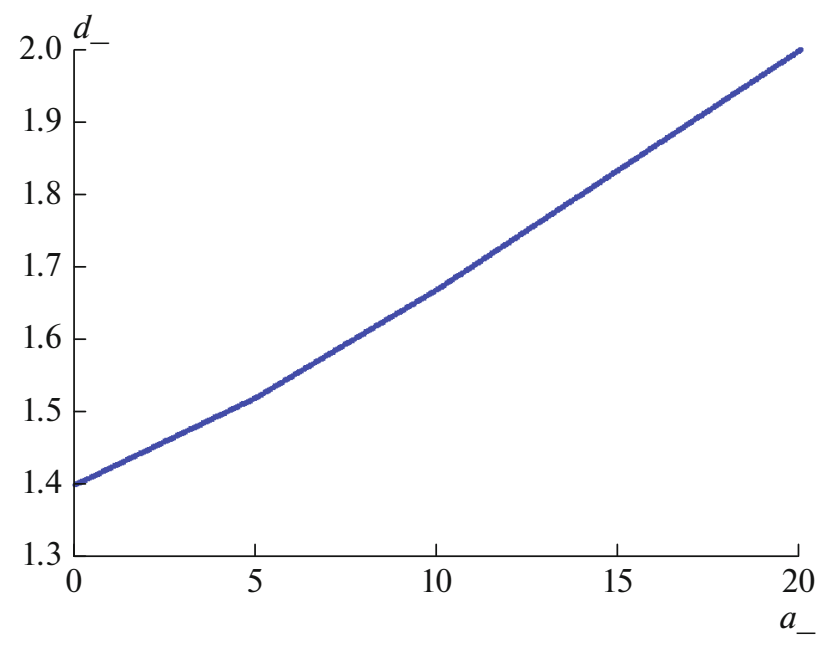

Fig. 5. Dependence of position $d_{-}$of the interferogram derivative maximum on the target slit of width $a_{-}$in relative units.

with an electron energy of $6.1 \mathrm{MeV}$ and a frequency of $8 \mathrm{~Hz}$. A macropulse of accelerated electrons with a duration of $4 \mu$ s is a sequence of $\approx 1.6 \times 10^{4}$ electron bunches. The population of the bunch was $\approx 7 \times 10^{7}$, which corresponded to the average current in a macro pulse of $30 \mathrm{~mA}$. Under these conditions, the emission of all electrons in a bunch at wavelengths exceeding $8 \mathrm{~mm}$ is coherent. The upper limit of $\lambda_{\mathrm{c}}=26 \mathrm{~mm}$ is determined by the out-of-limit waveguide, which was used to suppress the background from the high-frequency system of the accelerator. The lower limit is determined by the form factor of the electron bunch, which depends on its longitudinal size. To register the radiation, a DP21-M broadband detector manufactured by the Tomsk NIIPP with a sensitivity of $0.3 \mathrm{~V} / \mathrm{mW}$, operating at room temperature, was used. The spectral range of the sensitivity of the detector specified by the manufacturer is $\lambda=4-35 \mathrm{~mm}$.

To eluminate the prewave zone effect, the angular distribution of radiation was measured with a parabolic telescope according to the technique described and tested in [15]. The detector is mounted in the focus of a parabola (Fig. 6) placed on a 440-mm-long rod, which rotates around an axis passing through the center of the target slit. In this case, the angular characteristics of radiation correspond to the characteristics measured in the far-wave zone and are comparable with the calculations in this approximation.

As a target, we used two plates made of a foil-clad fiberglass forming a $10-\mathrm{mm}$-wide gap between them. To change the shift $d$ between the plates, one of the plates was moved along the electron beam direction. The device for measuring the electron bunch length is made in the form of two units, which are installed on the path of the extracted beam to measure the length of electron bunches. The first unit consists of targets

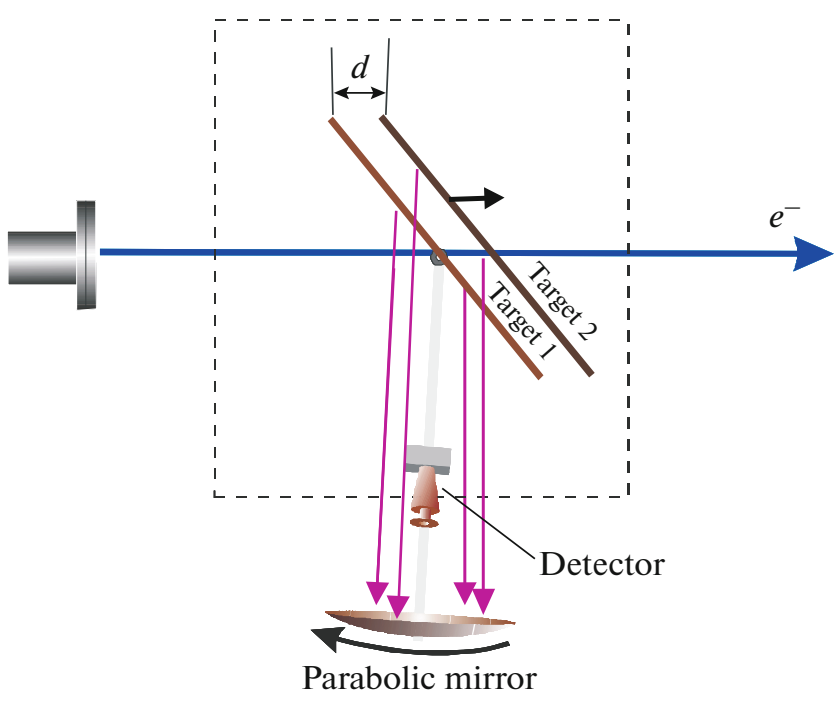

Fig. 6. Scheme of measuring the length of electron bunches.

rotated at an angle of $45^{\circ}$ with a device for moving one of them, the second unit is a parabolic telescope with a millimeter-wave detector for recording radiation.

\subsection{Results of Measurements}

When performing measurements, displacement $d=0$ was initially set (i.e., both plates were located in the same plane). In this position, the angular distribution of the horizontal polarization component of the diffraction radiation from the target was measured (Fig. 7), after which the telescope observation angle was set to the angular distribution minimum. The statistical error in measuring the radiation intensity was $6 \%$.

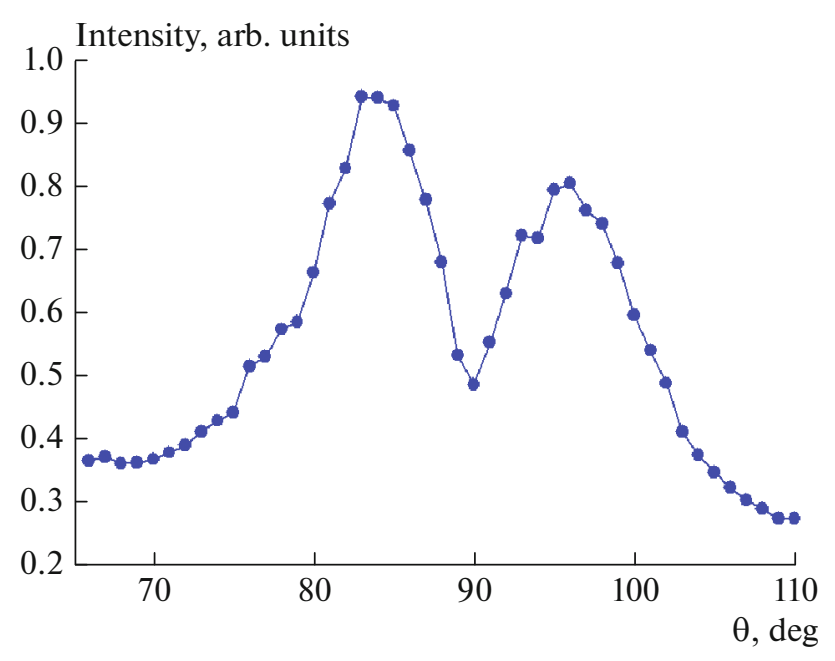

Fig. 7. Experimental angular distribution of diffraction radiation from a slit target. 


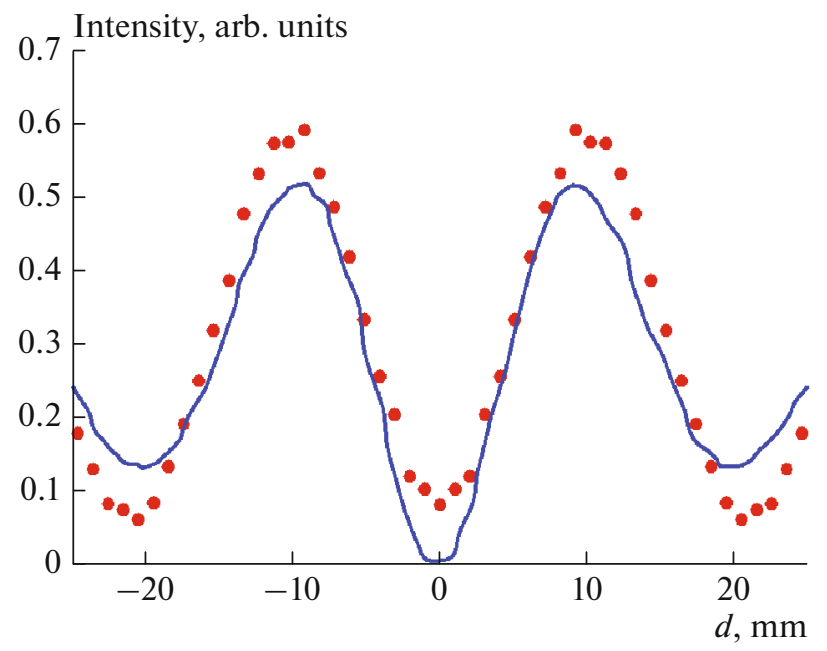

Fig. 8. Interferogram obtained by measuring the radiation intensity depending on the shift $d$. Points: experiment, solid line: calculation for experimental conditions.

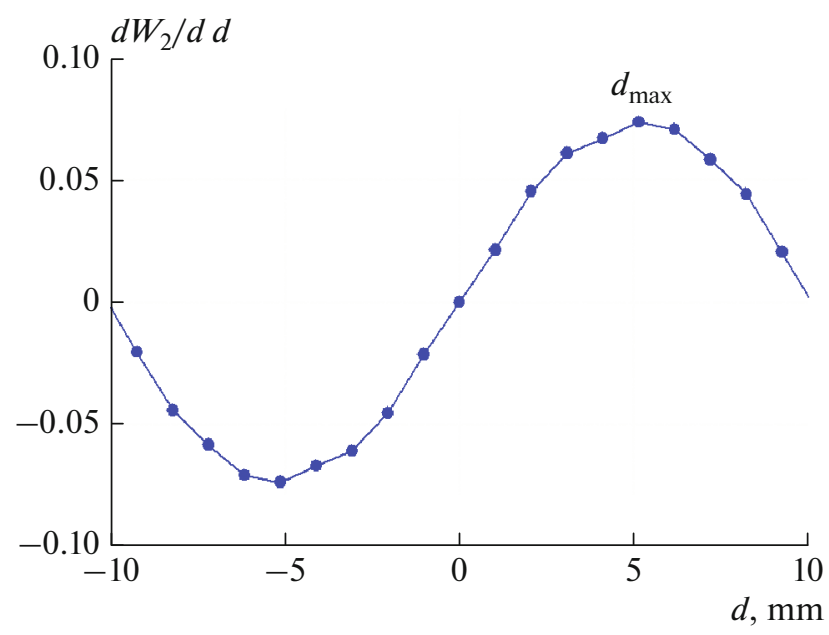

Fig. 9. Derivative in the region of the interferogram center shown in Fig. 8.

Then the system was ready to measure the longitudinal size of electron bunches. In this case, when recording interferograms, we measured the vertical component of the radiation polarization.

Figure 8 plots the interferogram obtained by measuring the dependence of the radiation intensity on the shift $d$ and the calculation for the experimental conditions.

The derivative of this interferogram in the region of its center $(d=0 \mathrm{~mm})$ is shown in Fig. 9. The position $d_{\max }$ of the derivative maximum is $5 \mathrm{~mm}$. To determine the electron bunch length, we use the dependence of the position of the maximum of the derivative as a function of $\sigma$, obtained from expression (1) for the experimental conditions (Fig. 10).

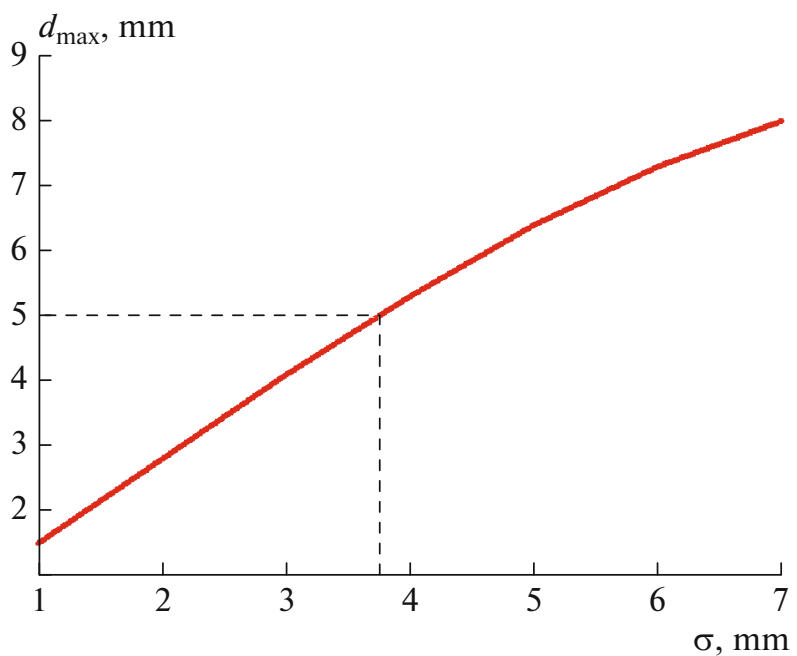

Fig. 10. Derivative maximum position as a function of $\sigma$ obtained from expression (1) for experimental conditions.

An assessment of the electron bunch lengths from these data yields $\sigma=3.7 \pm 0.3 \mathrm{~mm}$.

\section{CONCLUSIONS}

Thus, in this work we demonstrated the possibility of measuring the length of electron bunches in the millimeter range without disturbing the electron beam using a simple and affordable device in which the target of diffraction radiation acts as an interferometer. The chosen criterion for evaluating the electron bunch length provides, in comparison with [13], a sensitivity criterion of $25 \%$ (instead of $2.4 \%$ ) for $0.5 \mathrm{~mm}$ of the bunch length (see Fig. 10). The measured length of electron bunches satisfactorily agrees with the results obtained by other methods (for example, in [13]). In a stationary option, a parabolic telescope can be replaced by a parabolic reflector with a detector mounted at a fixed angle; i.e., the whole device can be mounted in one unit.

When measuring the length of submillimeter bunches $(\sigma<300 \mu \mathrm{m})$, the step of displacement of one target plate should not exceed $\Delta d \approx \sigma / 10$, which is quite feasible with commercially available piezodrives.

\section{ACKNOWLEDGMENTS}

This work was performed as part of the Program to Improve Competitiveness of Tomsk Polytechnical University.

\section{REFERENCES}

1. B. E. Carlsten and S. J. Russell, Phys. Rev. E 53, R2072 (1996).

2. H. Wiedemann, Particle Accelerator Physics (Springer, Heidelberg, 1993). 
3. Y. Shibata, T. Takahashi, T. Kanai, K. Ishi, and M. Ikezawa, Phys. Rev. E 50, 785 (1994).

4. V. Schlott, H. Loos, H. Gentz, et al., Part. Accel. 52, 45 (1996).

5. T. Watanabe, J. Sugahara, T. Yoshimatsu, et al., Nucl. Instrum. Methods Phys. Res., Sect. A 480, 315-327 (2002).

6. B. Feng, M. Oyamada, F. Hinode, et al., Nucl. Instrum. Methods Phys. Res., Sect. A 475, 492-497 (2001).

7. M. Castellano and V. A. Verzilov, Phys. Rev. E 63, 056501 (2001).

8. M. C. Lampel, Nucl. Instrum. Methods Phys. Res., Sect. A 385, 19 (1997).

9. D. C. Nguyen, Nucl. Instrum. Methods Phys. Res., Sect. A 393, 514-518 (1997).
10. D. C. Nguyen, Los Alamos National Laboratory, Los Alamos, NM.

11. S. J. Smith and E. M. Pursell, Phys. Rev. 92, 1069 (1953).

12. B. N. Kalinin, D. V. Karlovets, A. S. Kostousov, et al., Nucl. Instrum. Methods Phys. Res., Sect. B 252, 6268 (2006).

13. D. A. Shkitov, G. A. Naumenko, M. V. Shevelev, A. P. Potylitsyn, H. Deng, and X. Wang, J. Surf. Invest.: X-ray, Synchrotr. Neutron Tech. 7, 784-787 (2013).

14. A. P. Potylytsin, Nucl. Instrum. Methods Phys. Res., Sect. B 145, 169-179 (1998).

15. B. N. Kalinin, G. A. Naumenko, A. P. Potylitsyn, G. A. Saruev, L. G. Sukhikh, and V. A. Cha, JETP Lett. 84, 110 (2006). 